\title{
External Stenting of Vein Grafts in Coronary Artery Bypass Grating: Interim Results from a Two Centers Prospective Study
}

Luca Weltert ( $\sim$ lweltert@gmail.com )

European Hospital, Rome https://orcid.org/0000-0001-6094-8280

Katia Audisio

Azienda Ospedaliera Ordine Mauriziano di Torino

Alessandro Bellisaro

European Hospital

Gianluca Bardi

Azienda Ospedaliera Ordine Mauriziano di Torino

Roberto Flocco

Azienda Ospedaliera Ordine Mauriziano di Torino

Ruggero De Paulis

European Hospital

Paolo Centofanti

Azienda Ospedaliera Ordine Mauriziano di Torino

\section{Research article}

Keywords: Coronary artery bypass grafting, myocardial revascularization, vein grafts, vein graft disease, venous external stent

Posted Date: August 24th, 2020

DOI: https://doi.org/10.21203/rs.3.rs-62859/v1

License: (c) (1) This work is licensed under a Creative Commons Attribution 4.0 International License.

Read Full License

Version of Record: A version of this preprint was published at Journal of Cardiothoracic Surgery on April 12th, 2021. See the published version at https://doi.org/10.1186/s13019-021-01406-0. 


\section{Abstract}

Background - Previous studies evaluating external stents for saphenous vein grafts (SVG) in CABG were limited to on-pump isolated CABG and single grafting technique with one external stent per patient. The objective of this prospective study was to evaluate the safety and the short-term performance of external stents in heterogeneous group of patients which underwent on and off-pump CABG, single and sequential grafting.

Methods - 102 patients undergoing CABG were enrolled in two centers. All patients received internal mammary artery to the left anterior descending artery and additional arterial and/or venous grafts. In each patient, at least one SVG was supported with an external stent. Grafts' patency and SVG lumen uniformity were assessed using CT angiography at a pre-defined time window of 6-12 months post procedure. All patients were prospectively followed-up via phone call and/or visit every 6 months for Major Adverse Cardiac and Cerebrovascular Events.

Results - 51 patients (50\%) underwent off-pump CABG and 23 patients (23\%) were grafted with bilateral internal mammary arteries. Each patient received one or more SVG grafted in a sequential technique $(44 \%)$ or as single graft (56\%). All SVG were externally stented in $84 \%$ of patients and in $16 \%(n=16)$ one SVG was stented and one remained unsupported. At 6-12 months, patency rates of LIMA, RIMA, externally stented SVG and none-stented SVG were $100 \%, 100 \%, 98 \%$ and $87.5 \%$ respectively. $90 \%$ of the externally stented SVG had uniform lumen compared to $37 \%$ of the non-stented SVG. Clinical follow-up was completed for all patients with a mean duration of 20 months (range 6-54 months). During follow up period, one patient experienced myocardial infarction due to occlusion of the LIMA-LAD graft and one patient experienced transient ischemic attack.

Conclusions - External stenting of SVG is feasible and safe in CABG setting which includes off pump CABG and sequential SVG grafting and associated with acceptable early patency rates.

Study registration- Study was registered at ClinicalTrials.gov. NCT01860274 (initial release 20.05.2013)

\section{Introduction}

Coronary artery bypass grafting $(C A B G)$ remains the gold standard treatment for multivessel coronary artery disease (1). Despite extensive clinical research, the long-term outcome of CABG is still limited by the poor longevity of saphenous vein grafts (SVG), the most commonly used type of conduit (2). Although the root causes of SVG disease were discovered decades ago, these findings were not translated into the clinical setting in which only $50 \%$ of vein grafts are patent 10 years after CABG (3).

The accelerated SVG disease is attributed to early structural remodeling of the vein due to exposure to the hemodynamics of the arterial circulation and the development of intimal hyperplasia (4-5). Except for statins and beta blockers, pharmacological attempts to mitigate vein graft disease have shown limited success (6). Surgical approaches to reduce SVG disease, which were focused on optimization of the 
harvesting method, have shown that a no-touch technique leads to significant increase in SVG patency compared to conventional harvesting (7).

Experimental studies demonstrated significant effect of external stenting on the progression of vein graft disease post implantation (8-9). These findings were translated recently into the clinical setting and randomized trials have shown promising evidence regarding the effect of external stents on intimal hyperplasia and structural SVG remodeling (Fitzgibbon classification) up to 4.5 years after CABG (1013).

However, previous reports regarding the benefits of external stenting were limited to a highly selective group of patients which underwent isolated on-pump CABG and single SVG grafting. The objective of our study was to evaluate the safety, technical feasibility and early outcome of external stenting in routine practice which includes heterogenous group of patients undergoing on and off-pump CABG, single and sequential SVG grafting.

\section{Methods}

\section{Patients}

Patients undergoing elective surgical revascularization at two centers with at least one SVG were included in the study. Upon device availability in the hospital, consecutive patients were recruited by two senior surgeons (one in each hospital) who were trained with the implantation technique. The study was approved by the local ethics committee and all patients gave informed consent (NCT01860274). All patients underwent CABG with internal mammary artery (IMA) to the left anterior descending artery (LAD) and additional arterial or venous grafts. In each patient, at least one SVG was supported with an external stent (VEST, Vascular Graft Solutions, Israel) according to surgeon's discretion.

\section{Intraoperative}

In all cases, SVG harvesting was performed in a conventional open manner and all side branches were ligated with sutures or ties. Heparin was not given prior to SVG harvesting and the vein was stored in normal saline. During preparation, the vein was marked each $5 \mathrm{~cm}$ and its diameter was assessed using a dedicated measurement tool. After completion of the distal anastomosis, SVG length was accurately measured and an external stent model was chosen based on SVG length and diameter. The device was threaded over the vein and was manually expanded to cover the entire SVG leaving 2-10 mm unsupported segment near the anastomoses. In case of multiple distal anastomoses, SVG length and diameter of each segment was assessed after completion of the distal anastomosis and the appropriate stent model was chosen to support the segment between the two distal anastomoses (Fig. 1). During offpump CABG, if the SVG was dilated due to backflow after completion of the distal anastomosis, a vascular clamp was applied on the distal end of the SVG to allow deflation and enable stent expansion on a non-pressurized graft. The stent has radial elasticity and axial plasticity that enabled the surgeon to compress the stent in order to enable adequate evaluation/revision of the graft or the anastomosis with 
subsequent re-expansion. Prior to closing the chest, Transit Time Flow Measurement (TTFM) was applied to all grafts and corrective measures were taken as needed. In order to perform TTFM measurements, the proximal end of the stent was slightly compressed and then re-positioned after completion of TTFM measurement.

Assessment and optimization of postoperative coagulation state were performed using Point of Care Thromboelastography. After CABG, all patients were prescribed with statins, anti-platelets and beta blockers. In-hospital adverse events were recorded including death, myocardial infarction, stroke, transient ischemic attack and need for revascularization.

\section{CT angiography}

CT angiography (OPTIMA 660, GE, 64 slices) was performed to assess grafts patency and lumen uniformity 6-12 after CABG (Fig. 2). Oral nitroglycerin was given prior to the scan in order to achieve coronary vasodilatation; $\beta$-blocker (Esmololo, $1 \mathrm{fl}$ ) was also administered to patients with heart rate above $65 \mathrm{bpm}$. After defining the region of interest, $95 \mathrm{ml}$ of an iodine based contrast agent (Ultravist 370) was injected at a flow rate of $5 \mathrm{ml} / \mathrm{sec}$ followed by a saline chaser bolus of $40 \mathrm{~mL}$ at $5 \mathrm{ml} / \mathrm{sec}$, via a 20 gauge needle in the antecubital fossa. The gantry rotation time was $0.35 \mathrm{sec}$, peak tube voltage was $120 \mathrm{kVp}$, and current ( $\mathrm{mA}$ ) was adjusted per patient's body weight. SVG were graded by an independent observer (radiologist) to be (1) patent with $<50 \%$ stenosis (2) patent with $>50 \%$ stenosis (3) occluded. In addition, as shown in Fig. 3, SVG were classified to have uniform lumen or lumen irregularities defined as lumen diameter variation $>0.5 \mathrm{~mm}$.

\section{Clinical follow up}

All patients will be prospectively followed-up via phone call and/or visit every 6 months for a period of 5 years. Major Adverse Cardiac and Cerebrovascular Events (MACCE), defined as the composite occurrence of all-cause mortality, myocardial infarction, revascularization, and stroke are recorded per each phone call or visit.

\section{Statistical analysis}

Continuous data are expressed as mean and standard deviations or median and range after assessment of normality distribution. Categorical data are presented as absolute values and percentages. Assessment of normality of distribution was performed by Kolmogorov-Smirnov test.

\section{Results}

Between September 2015 and December 2019, 102 patients that underwent CABG were enrolled to this study. None of the patients had prior cardiac surgery and $26 \%$ of patients had prior percutaneous revascularization. Baseline demographics are presented in Table 1. All patients were maintained on aspirin during CABG and $96 \%$ of patients were receiving statins (20 mg Atorvastatin) at the time of operation. 
Table 1

- Baseline demographics

\begin{tabular}{|ll|}
\hline & Mean \pm SD or n (\%) \\
\hline Age (yrs.) & $68.5 \pm 11$ \\
\hline Gender (male) & $89(87 \%)$ \\
\hline Weight (kg) & $74 \pm 12$ \\
\hline Height (cm) & $168 \pm 7$ \\
\hline Body Mass Index (kg/cm2) & $26 \pm 4$ \\
\hline Ex smokers or current smokers & $84(82 \%)$ \\
\hline Insulin dependent diabetes & $28(27 \%)$ \\
\hline Hypertension & $86(84 \%)$ \\
\hline Dyslipidemia & $84(82 \%)$ \\
\hline Diffuse peripheral artery disease & $13(13 \%)$ \\
\hline Vasculitis & $0(0 \%)$ \\
\hline Prior cardiac surgery & $0(0 \%)$ \\
\hline Prior percutaneous revascularization & $27(26 \%)$ \\
\hline Prior stroke in the past 2 years & $6(6 \%)$ \\
\hline COPD of at least moderate grade & $23(23 \%)$ \\
\hline NYHA Class & $2.2 \pm 1.5$ \\
\hline CCS Class & $3 \pm 2.2$ \\
\hline Ejection Fraction & $55 \pm 7 \%$ \\
\hline Creatinin (mg/dl) & $1.07 \pm 0.7$ \\
\hline Euroscore & $3.6 \% \pm 3.8 \%$, \\
\hline & \\
\hline
\end{tabular}

Revascularization strategy was determined by the surgeon and no change in the surgical plan was made intraoperatively due to the use of the external stent. 51 patients (50\%) underwent off-pump CABG. All patients underwent CABG with IMA-LAD grafts and 23 patients $(23 \%)$ received bilateral internal mammary artery (BIMA) grafting. In addition, each patient received one or more SVG grafted in a sequential technique with two or three segments $(n=45)$ or as a single conduit with one distal anastomosis $(n=57)$. In $84 \%$ of the patients $(n=86)$ all SVG were supported with an external stent and in $16 \%(n=16)$ at least one SVG was stented and one remained unsupported. Grafts distribution according to the coronary target is presented in Table 2. External stent deployment was successful in all patients with no technical failure. 
Expansion of the stent to cover the entire SVG length did not damage the anastomosis or caused new bleeding from the suture sites. In patients undergoing on-pump CABG warm intermittent blood cardioplegia was used with average cross-clamp time of $73 \pm 20$ minutes and average pump time of $97 \pm$ 24 minutes. Off-pump surgery was performed using the Medtronic Octopus Stabilizer (Medtronic, Dublin, Ireland) and FlowThru coronary shunts (Synovis, Birmingham, UK) of adequate size. 3 proximal anastomoses of SVG and 2 distal anastomoses of arterial grafts were revised due to abnormal TTFM readings (flow $<20 \mathrm{ml} / \mathrm{min}$ and/or $\mathrm{Pl}>5$ ). During revision of the venous grafts, the external stent was slightly compressed to enable graft exposure and was then re-expanded following revision. Intra and post-operative data is summarized in Table 3. 
Table 2

- Grafts distribution according to the coronary target.

\begin{tabular}{|ll|}
\hline & N (\%) \\
\hline LIMA graft & 118 \\
\hline LIMA to LAD & $99(83.8)$ \\
\hline LIMA to CRX & $0(0)$ \\
\hline LIMA to OM & $7(5.9)$ \\
\hline LIMA to Diagonal & $8(6.7)$ \\
\hline LIMA to IR & $4(3.3)$ \\
\hline RIMA graft & 23 \\
\hline RIMA to LAD & $3(13)$ \\
\hline RIMA to CRX & $0(0)$ \\
\hline RIMA to OM & $11(47.8)$ \\
\hline RIMA to Diagonal & $1(4.3)$ \\
\hline RIMA to IR & $8(34.7)$ \\
\hline EXternally stented SVG & 110 \\
\hline SVG to CRX & $11(10)$ \\
\hline SVG to OM & $28(25.4)$ \\
\hline SVG to diagonal & $16(14.5)$ \\
\hline SVG to IR & $11(10)$ \\
\hline SVG to PDA & $31(28.1)$ \\
\hline SVG to RCA & $13(11.8)$ \\
\hline None stented SVG & 16 \\
\hline SVG to CRX & $1(6.2)$ \\
\hline SVG to OM & $6(37.5)$ \\
\hline SVG to diagonal & $7(43.7)$ \\
\hline SVG to IR & $0(0)$ \\
\hline SVG to PDA & $2(12.5)$ \\
\hline SVG to RCA & \\
\hline
\end{tabular}


Table 3

- Intra and post-operative data

\begin{tabular}{|ll|}
\hline & $\mathbf{N}(\%)$ \\
\hline Concomitant valve or aortic surgery & $13(13 \%)$ \\
\hline Off-pump CABG & $51(50 \%)$ \\
\hline IMA-LAD grafts & $102(100 \%)$ \\
\hline Use of RIMA & $23(23 \%)$ \\
\hline Single vein graft (pts.) & $57(56 \%)$ \\
\hline Sequential vein grafts (pts.) & $45(44 \%)$ \\
\hline Mean surgery time (min.) & $187 \pm 37$ \\
\hline Mean cross clamp time (min.) & $73 \pm 20$ \\
\hline Mean pump time (min.) & $97 \pm 24$ \\
\hline Mean extubation time (h.) & $8.2 \pm 6$ \\
\hline Mean Intensive Care Unit Stay (days) & $1.6 \pm 1.12$ \\
\hline Mean in Hospital Stay (days) & $7.4 \pm 1.5$ \\
\hline
\end{tabular}

One patient experienced a perioperative myocardial infarction $(0.9 \%)$ due to occlusion of the Left Internal Mammary Artery (LIMA)-LAD graft and was treated by PCl. Of note, this patient had undergone endarterectomy of the LAD during operation. One patient experienced a transient ischemic attack on postoperative day 4 (0.9\%). No other in-hospital events were recorded.

All patients completed CTangiography assessment at 6-12 months' time window. As shown in Table 4, all the arterial grafts were patent. In addition, $98 \%$ of the externally stented SVG (2 occlusions) and $87.5 \%$ of the non-stented grafts (2 occlusions) were patent. No differences in SVG patency were observed between the off and on pump CABG groups or the different grafting techniques (single versus sequential). $90 \%$ of the externally stented SVG had uniform lumen compared to $37 \%$ of the non-stented SVG. No CTangiograohy artifacts were observed in the externally stented SVG and the lumen was well defined in all grafts. CTangiography imaging demonstrated well the spatial positioning of the SVG and the stents, especially in multi-anastomoses sequential setting, and no kinking or unexpected twisting were observed. 
Table 4

- Grafts patency and uniformity assessed by CT angoigrpahy

\begin{tabular}{|llll|}
\hline & $\mathbf{N}(\%)$ & $\mathbf{N}(\%)$ & $\mathbf{N}(\%)$ \\
\hline & Patent & Patent & Occluded \\
\hline LIMA-LAD (99) & $(<50 \%$ stenosis) & $(>50 \%$ stenosis) & \\
\hline RIMA (23) & $99(99 \%)$ & $1(1 \%)$ & \\
\hline Exteranlly stented SVG: & $23(100 \%)$ & & \\
\hline - Single SVG (65) & & \\
\hline - Sequential SVG (45) & $63(97 \%)$ & \\
\hline None stented SVG: & $45(100 \%)$ & \\
\hline - Single SVG (16) & & \\
\hline - Sequential SVG (0) & $14(87.5 \%)$ & \\
\hline SVG Uniformity at CT angiography: & & \\
\hline Exteranlly stented SVG (110) & & \\
\hline - Uniform lumen & $99(90 \%)$ & \\
\hline - Non-uniform lumen & $11(10 \%)$ & \\
\hline None Stented SVG (16) & & \\
\hline - Uniform lumen & & \\
\hline - Non-uniform lumen & & \\
\hline
\end{tabular}

Clinical follow-up was completed for all patients with a mean duration of 20 months (range 6-54 months). During the follow-up period no additional MACCE events were recorded.

\section{Discussion}

This report describes the use of external stents for SVG during routine CABG practice which includes off pump CABG, sequential grafting and the use of multiple external stents per patient. To the best of our knowledge, this is the first time that external stents performance is being evaluated as part of routine practice.Our main finding is that external stenting is safe and can be seamlessly integrated into the routine $C A B G$ practice, with minimal changes to the standard grafting technique. No traction on the anastomoses was observed during stent positioning and the ability to shape the device together with the graft in-situ allowed for precise positioning of the vein curvatures in multiple anastomoses sequential settings. 
The etiology of early SVG failure is multifactorial and related to surgical skill, graft quality, harvesting technique and the size of the coronary vascular bed (4-5). As shown in Table 5, contemporary trials reported early (up to 1 year after CABG) SVG patency rates between $80-85 \%$ in on pump CABG and less than $80 \%$ in off pump CABG (14-19). The accuracy of CT angiography in detecting the presence and severity of coronary and graft disease was shown to be comparable to invasive coronary angiography in several trials. Studies related to 64-slices CTangiography technology reported sensitivity values of 93$99 \%$ and specificity of $95-97 \%$ with a negative predictive value of $99 \%(20)$. One potential explanation for the high early patency rates observed in our study is the use of open rather than endoscopic harvesting technique. Recent meta analysis has shown that endoscopic vein harvesting, even in experienced hands, may reduce short and intermediate term SVG patency due to trauma to the vein and endothelial damage (21). In addition, all procedures were performed by senior surgeons who used TTFM to identify technical errors prior closing the chest. As previously reported and confirmed also in our study, TTFM allows detection of technical problems at the level of the anastomoses, leading to the revision of $2-4 \%$ of bypass grafts with a consequent reduction in early graft failure and its related clinical events (22). Our Iow MACCE rates may be attributed in part to the high early patency rates of the venous and the arterial grafts. As shown in the PREVENT IV trial, 18 months after CABG, death, myocardial infarction and revascularization rate were significantly higher in patients who experienced at least one early SVG failure compared to those who didn't ( $26 \%$ compared to $1.8 \%$ respectively) (16).

Table 5

- SVG patency rates at 1 year

\begin{tabular}{|c|c|c|c|}
\hline & \# of patients & \# of vein grafts & SVG Patency rate at 1 year (\%) \\
\hline Desai et al $(2004)^{21}$ & 440 & 440 & 86.4 \\
\hline Goldman et al $(2004)^{22}$ & 799 & & 84 \\
\hline PREVENT IV $(2005)^{23}$ & 951 & 2242 & 73.5 \\
\hline Cho et al $(2006)^{24}$ & 109 & 227 & 82.4 \\
\hline Kim et al $(2008)^{25}$ & 349 & 121 & 76 \\
\hline \multirow[t]{4}{*}{ Shroyer et al $(2009)^{26}$} & 685 & 1262 & 76.6 \\
\hline & & Off-pump & \\
\hline & 686 & 1339 & 83.8 \\
\hline & & On-pump & \\
\hline
\end{tabular}

The majority of externally stented SVGs demonstrated uniform lumen. This finding is in correlation with previous pre-clinical and clinical reports on the beneficial effect of external stents on lumen uniformity 
flow patterns and the protective effect of laminar flow against the development of SVG intimal hyperplasia (10-13). This is in contrast to areas with low and oscillatory wall shear stress which are more prone to endothelial dysfunction, thrombus formation and more aggressive vascular disease (23).

Our study is part of intensive clinical research aiming to overcome the Achilles heel of CABG. SVG preservation solutions, no-touch harvesting technique and external stents are all attempts to change the natural history of SVG. Even if it does not immediately translate to hard clinical endpoints, mitigating well validated surrogate SVG disease markers such as intimal hyperplasia and lumen irregularities is another important step in the journey to improve the clinical outcome of surgical revascularization.

This study has single arm with relatively low sample size and limited follow up duration. The lack of proper randomization between the stented and non-stented SVGand the fact that two experienced coronary surgeons performed all the procedures, limit the generalizability of the results. Larger, well designed, randomized trials with 5-10 years follow up are required to further define the role of external stents in $C A B G$ and its clinical benefit.

\section{Conclusions}

The use of external stents in a complex, 'real world' CABG setting is technically feasible and safe with acceptable early patency rates and good clinical outcome. According to our experience, the device can be integrated into routine CABG practice with minimal learning curve.

\section{Abbreviations}

SVG

Saphenous Vein Graft

CABG

Coronary Artery Bypass Grafting

MACCE

Major Adverse Cardiac and Cerebrovascular Events

IMA

Internal Mammary Artery

LAD

Left Anterior Descending Artery

LIMA

Left Internal Mammary Artery

RIMA

Right Internal Mammary Artery

BIMA

Bilateral Internal Mammary Artery

TTFM 


\section{Declarations}

\section{Ethics approval and consent to participate}

The study was approved by the ethics committee of GHC Healthcare (progressive number 2018-002). All patients gave informed consent.

Consent for publication-not applicable.

\section{Availability of data and materials}

The datasets used and/or analysed during the current study are available from the corresponding author on reasonable request

\section{Competing interests}

The authors declare that they have no competing interests.

\section{Funding}

This research did not receive any funding.

\section{Authors' contributions}

LPW-Study Conception and design, data collection, data analysis, writing the manuscript.

KA- Data collection, writing the manuscript.

AB- Writing the manuscript.

GB- Writing the manuscript.

RF- Writing the manuscript.

RDP- Writing the manuscript.

PC-Study conception and design, data collection, data analysis, writing the manuscript.

All authors reviewed and approved the final manuscript.

\section{Acknowledgements}

Not applicable. 


\section{References}

1. Neumann FJ, Sousa-Uva M, et al. 2018 ESC/EACTS Guidelines on myocardial revascularization: The Task Force on Myocardial Revascularization of the European Society of Cardiology (ESC) and the European Association for Cardio-Thoracic Surgery (EACTS). Developed with the special contribution of the European Association for Percutaneous Cardiovascular Interventions (EAPCI). Eur Heart J. 2018;00:1-96.

2. $10.1053 /$ j.semtcvs.2017.05.018

Schwann TA, Tatoulis J, Puskas J, Bonnell M, Taggart DP, Kurlansky P, Jacobs JP, Thourani VH, O'Brien S, Wallace A, Engoren MC, Tranbaugh RF, Habib RH. Worldwide Trends in Multi-arterial Coronary Artery Bypass Grafting Surgery 2004-2014: A Tale of 2 Continents. Semin Thorac Cardiovasc Surg. 2017 Autumn;29(3):273-280. doi: 10.1053/j.semtcvs.2017.05.018.

3. Gaudino M, Benedetto U, Fremes S, Biondi-Zoccai G, Sedrakyan A, Puskas JD, Angelini GD, Buxton B, Frati G, Hare DL, Hayward P, Nasso G, Moat N, Peric M, Yoo KJ, Speziale G, Girardi LN, Taggart DP. RADIAL Investigators. Radial-Artery or Saphenous-Vein Grafts in Coronary-Artery Bypass Surgery. N Engl J Med. 2018 May;31(22):2069-77. doi:10.1056/NEJMoa1716026. 378 ) .

4. Harskamp RE, Lopes RD, Baisden CE, de Winter RJ, Alexander JH. Saphenous vein graft failure after coronary artery bypass surgery: pathophysiology, management, and future directions. Ann Surg. 2013;257:824-33.

5. Motwani JG, Topol EJ. Aortocoronary Saphenous Vein Graft Disease: Pathogenesis, Predisposition, and prevention. Circulation. 1998;97:916-31.

6. Une D, Kulik A, Voisine P, Le May M, Ruel M. Correlates of saphenous vein graft hyperplasia and occlusion 1 year after coronary artery bypass grafting: analysis from the CASCADE randomized trial. Circulation. 2013 Sep 10;128:(S213-S218).

7. Samano N, Geijer $\mathrm{H}$, et al. The no-touch saphenous vein for coronary artery bypass grafting maintains a patency, after 16 years, comparable to the left internal thoracic artery: a randomized trial. J Thorac Cardiovasc Surg. 2015;150:880-8.

8. Hu J, Wan S. External support in preventing vein graft failure. Asian Cardiovasc Thorac Ann. 2012;20(5):615-22.

9. Jeremy JY, Gadsdon P, et al. On the biology of saphenous vein grafts fitted with external synthetic sheaths and stents. Biomaterials. 2007;28:895-908.

10. Taggart DP, Ben Gal Y, et al. A randomized trial of external stenting for saphenous vein grafts in coronary artery bypass grafting. Ann Thorac Surg. 2015;99:2039-45.

11. Mario C. OCT imaging

Webb CM, Orion E, Taggart DP, Channon KM Di. Mario C. OCT imaging.

12. of aorto-coronary. vein graft pathology modified by external stenting:1-year post-surgery. Eur Heart $\mathrm{J}$ Cardiovasc Imaging. 2016;17:1290-5. 
13. Meirson T, Orion E, Di Mario C, Webb C, Patel N, Channon KM, et al. Flow patterns in externally stented saphenous vein grafts and development of intimal hyperplasia. J Thorac Cardiovasc Surg. 2015;150:871-9.

14. Taggart DP, Webb CM, Desouza A, Yadav R, Channon KM, De Robertis F, Di Mario C. Long-term performance of an external stent for saphenous vein grafts: the VEST IV trial. J Cardiothorac Surg. 2018 Nov 19;13(1):117. doi: 10.1186/s13019-018-0803-9.

15. Desai N, Cohen E, Naylor C, Fremes S, Investigators R. A Randomized Comparison of Radial-Artery and Saphenous-Vein Coronary Bypass Grafts New England. J Med. 2004;351(22):2302-9.

16. Goldman S, Zadina K, Moritz T, Ovitt T, Sethi G, Copeland J, Thottapurathu L, Krasnicka B, Ellis N, Anderson R, Henderson W, \#207/297/364, V. (2004). Long-term patency of saphenous vein and left internal mammary artery grafts after coronary artery bypass surgery Journal of the American College of Cardiology 44(11), 2149-2156.

17. Alexander JH, Hafley G, Harrington RA, Peterson ED, Ferguson TB Jr, Lorenz TJ, Goyal A, Gibson M, Mack MJ, Gennevois D, Califf RM, Kouchoukos NT. PREVENT IV Investigators. Efficacy and Safety of Edifoligide, an E2F Transcription Factor Decoy, for Prevention of Vein Graft Failure Following Coronary Artery Bypass Graft Surgery. JAMA. 2005;294:2446-54.

18. Cho K, Kim J, Jae-Sung C, Kim K. Serial angiographic follow-up of grafts one year and five years after coronary artery bypass surgery. Eur J Cardiothorac Surg. 2006;29(4):511-6.

19. Kim K, Cho K, Jeong D. Midterm angiographic follow-up after off-pump coronary artery bypass: Serial comparison using early, 1-year, and 5-year postoperative angiograms. The Journal of Thoracic Cardiovascular Surgery. 2008;135(2):300-7.

20. Shroyer L, Grover F, Hattler B, Collins J, McDonald G, Kozora E, Lucke J, Baltz J, Novitzky D, Group V. On-Pump versus Off-Pump Coronary-Artery Bypass Surgery The New England. J Med. 2009;361(19):1827-37.

21. Mowatt G, Cook JA, Hillis GS, et al. 64-Slice computed tomography angiography in the diagnosis and assessment of coronary artery disease: systematic review and metaanalysis. Heart. 2008;94(11):1386-93.

22. Li G, Zhang Y, Wu Z, Liu Z, Zheng J. Mid-term and long-term outcomes of endoscopic versus open vein harvesting for coronary artery bypass: A systematic review and meta-analysis. Int J Surg. 2019 Dec;72:167-73. doi:10.1016/j.ijsu.2019.11.003.

23. Intraoperative transit-time. flow measurement and high-frequency ultrasound assessment in coronary artery bypass grafting. Taggart, David P. et al. The Journal of Thoracic and Cardiovascular Surgery, Volume 159, Issue 4, 1283-1292.e2.

24. Chiu JJ, Chien S. Effects of Disturbed Flow on Vascular Endothelium: Pathophysiological Basis and Clinical Perspectives. Physiol Rev. 2011;91:327-87.

\section{Figures}



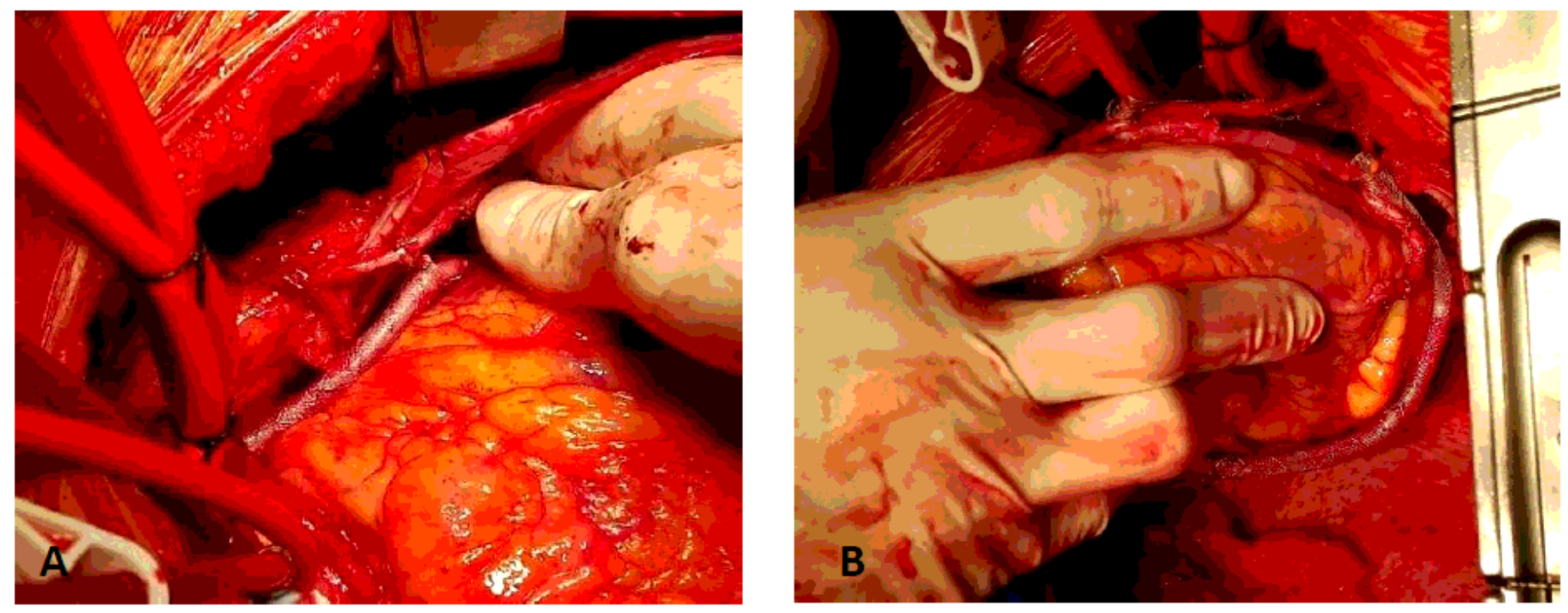

Figure 1. VEST implantation; A. sequential SVG bypassed to the Posterior Descending (PDA). B. AVG bypassed to the Ramus Intermediate (RI)

\section{Figure 1}

VEST implantation (A) sequential SVG bypassed to the posterior descending artery (B) aortocoronary SVG segment bypassed to the ramus intermediate artery.

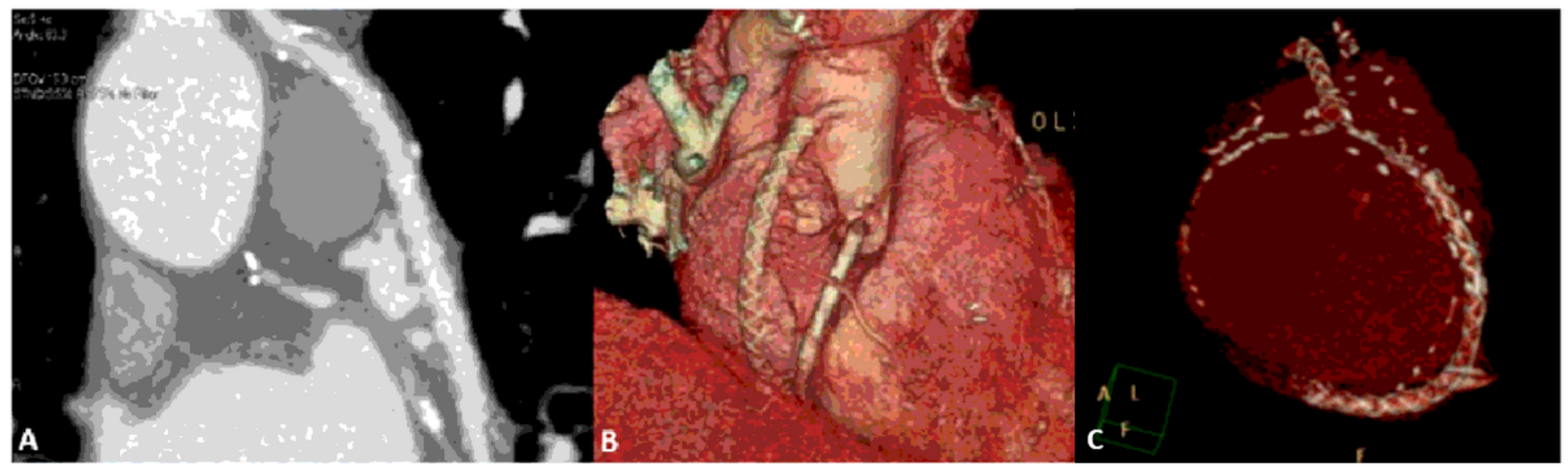

\section{Figure 2}

CT angiography of externally stented SVG to the right coronary artery in a 2D (A) and 3D reconstruction (B), and a 3D reconstruction of externally stented sequential SVG to the ramus intermediate and the posterior descending arteries (C). 

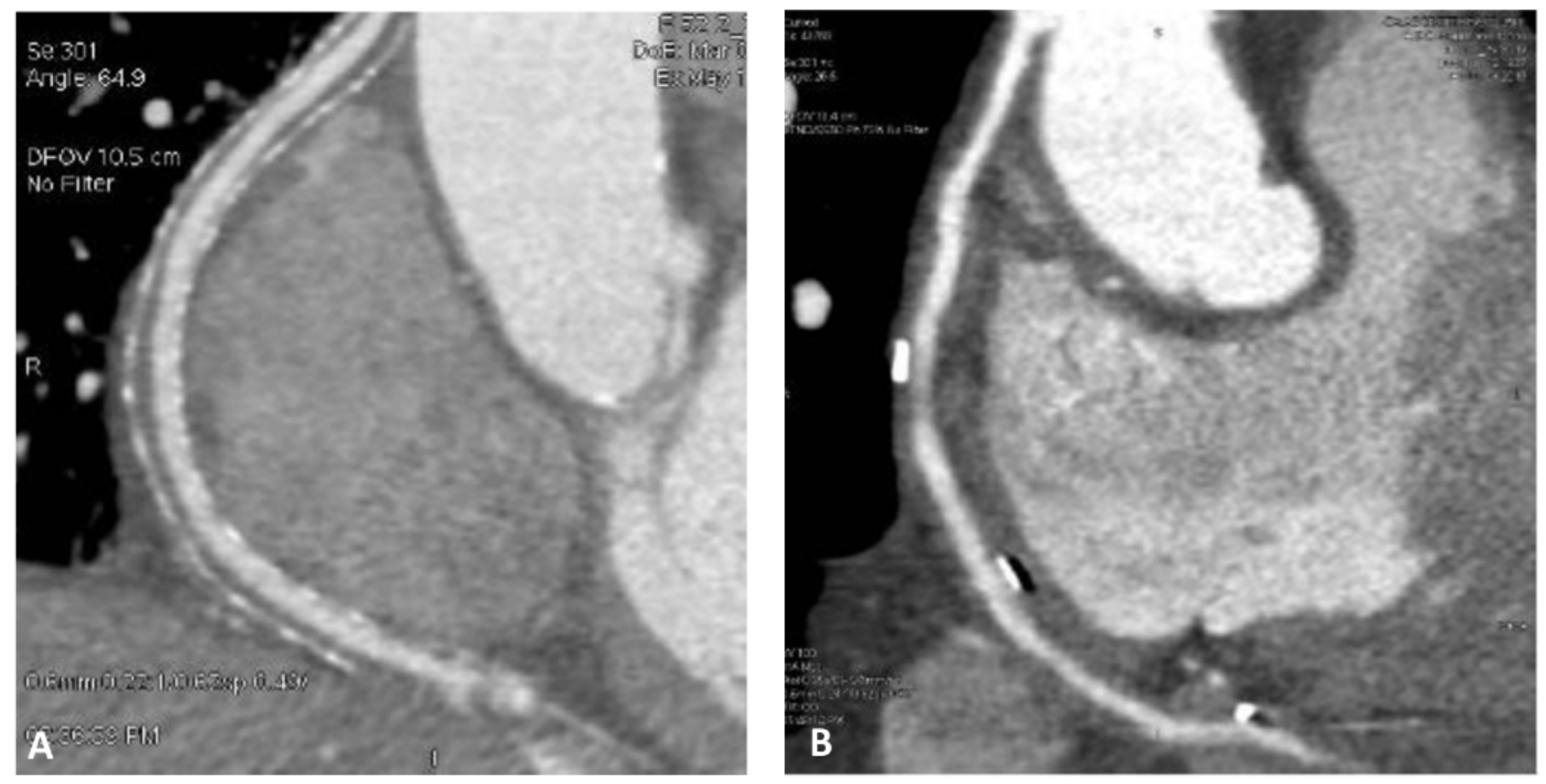

\section{Figure 3}

Extenally stented SVG to right coronary territory demonstrating a uniform lumen (A) and unsupported SVG to the right coronary territory with a non-unifrom lumen (B) 